\title{
Programming Mobile Applications
}

\section{Nada N. Saleem \\ Nada_N_S@uomosul.edu.iq \\ Mohammad Abdalraheem Hamdy}

College of Computer Sciences and Mathematics

University of Mosul, Mosul, Iraq

Received on: 01/12/2011

Accepted on: 19/04/2012

\section{ABSTRACT}

Mobile and wireless devices become widespread devices in the past few years, These devices made substantial progress in the important field of wireless applications which used in all areas of human life. This research develops two applications which execute on mobile and computer. The first application is electronic library which make the user search in the library electronically on computer throw Bluetooth wireless technology, The second application is mouse controlling throw Bluetooth.

These applications are programmed with J2ME language on mobiles, covered MIDP 2.0 with CLDC 1.0 executed on mobiles with versions Nokia 6600, Nokia 7610, N72 and executed on computers with the following operating systems Windows 7 , Windows XP and Linux Mandriva 2010.

Keyword: mobile, Bluetooth, wireless technology

$$
\text { ندى نعت سليم }
$$$$
\text { تاريخ استلام البحث: 2011/12/01 }
$$

\section{الملخص}

أصبحت أجهزة النقال والأجهزة اللاسلكية من الأجهزة الواسعة الانتشار في السنوات القليلة الماضية، وقد

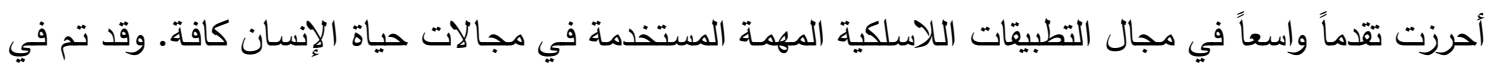
هذا البحث تصميم تطبيقين يعملان على عدد من أجهزة الموبايل والحاسوب، تتاول التطبيق الأول برنامجاً يسهل عملية البحث الكترونياً في المكتبة على أجهزة الحاسوب من خلال استخدام تقنية الـ Bluetooth, أمسا التطبيق الثاني فهو برنامج التحكم بفأرة الحاسوب من خلال جهاز الموبايل باستخدام تقنية الـ Bluetooth.

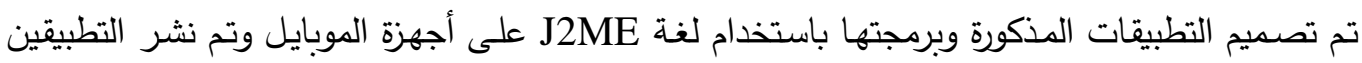

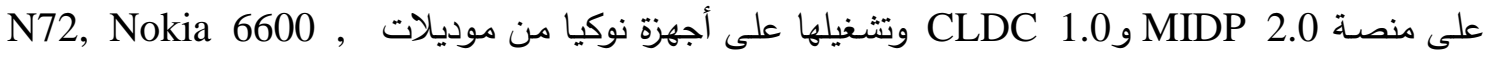

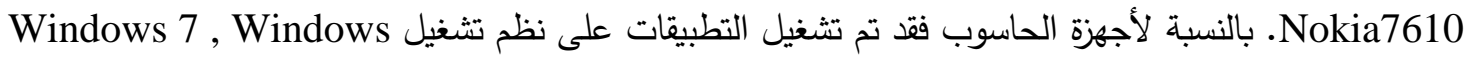
. Linux Mandriva ونظام تشغيل XP الكلمات المفتاحية: الموبايل, بلوتوث, التقنيات اللاسلكية

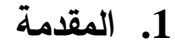
دخلت صناعة أجهزة المحمول والاتصـالات اللاسلكية في وقت قياسي، ومـع ازدياد الطلب على هذه

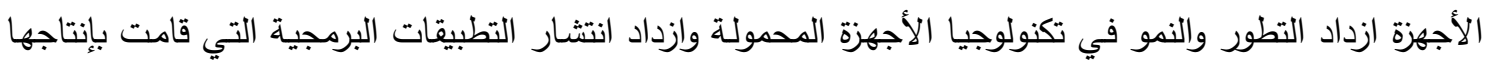
الثركات المختلفة والتي قدمت خدمات واسعة في عدة مجالات منها في مجال الأعمال والوصول إلى أي معلومة وفي أي مكان من خلال الاتصالات اللاسلكية. 
أصبحت أجهزة الموبايل بأحجام وأشكال مختلفة في الوقت الحاضر، ويتم اختيار الموبايل بالاعتماد على عدة معايير بالإضافة إلى الكلفة مثل : • جم الجهاز ووزنه.

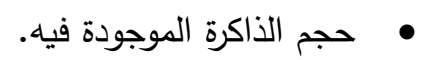

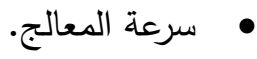

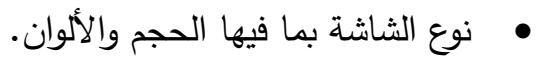

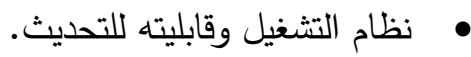

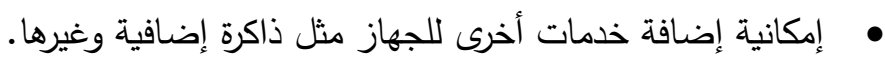

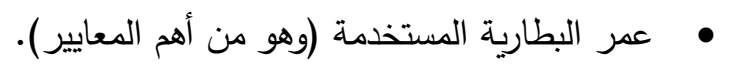

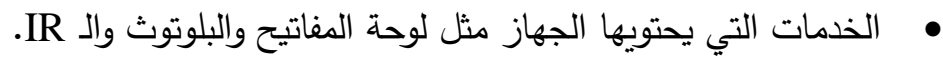

تعد هذه المعايير من الأمور المهمة التي يجب أخذها بنظر التقييم عند اختيار الموبايل والتي من الممكن الإطلاع

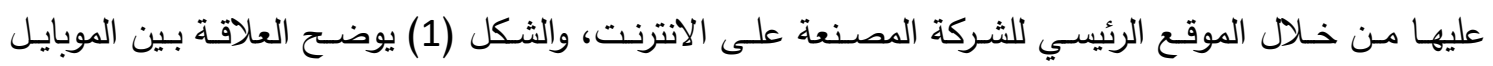

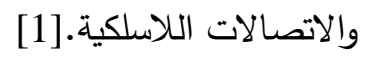

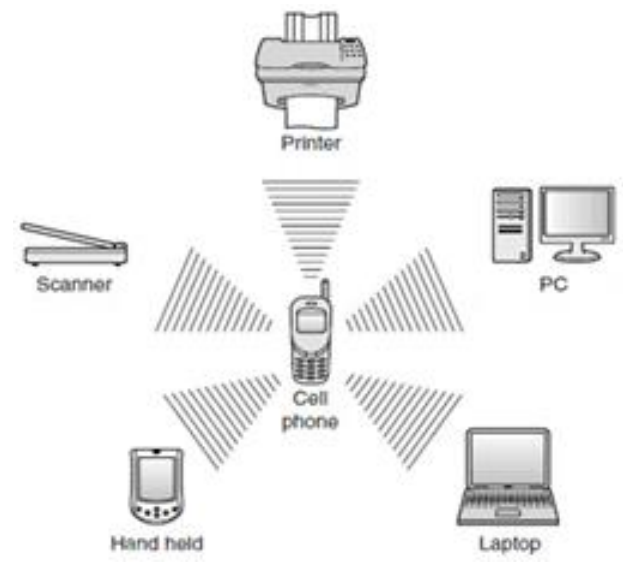

شكل (1) العلاقة بين الموبايل والاتصالات اللاسلكية [2]

وعند النظر في التطبيقات المستخدمة في أجهز الموبايل تدرجا من الأجهزة الاعتيادية البسيطة وصعودا إلى أجهزة الـ (IPhone) فبالإضـافة إلى اختلاف الهيكلية التي صدم بها كل تطبيق سوف نلاحظ الاختلاف في تفاعل المستخدم مـع كل تطبيق في هذه الأجهزة مع الأخذ بنظر التقييم الإمكانيات التي يقدمها كل جهاز ، فعلى سبيل المثال يكون التعامل مع التطبيقات في أجهزة الـ (IPhone) من خلال اللمس على شاشة الأنة الجهاز وبشكل مباشر .

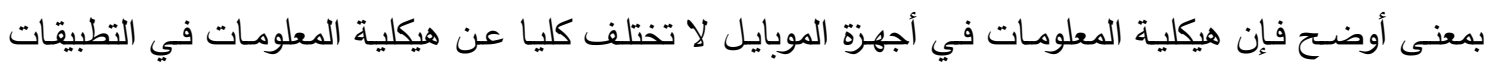

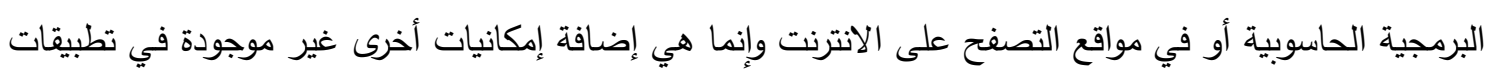
المجالات الأخرى.[3]

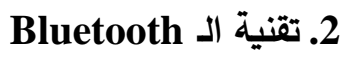

كانت بداية استخدام تقنية البلوتوث في عام 1994 عندما بدأت شركة Ericsson في دراسة لإيجاد وسائل بديلة لتأمين الاتصال بين أجزة الموبايل وملحقاتها، وهي تقنية مجانية ومتوفرة للجميع تؤمن الاتصال بمدى معين وطاقة 
مناسبة وكلفة أقل وتعمل على تردد 2.4 GHz وتستخدم حزمة الـ Industrial Scientific Medical (ISM) وهي حزمة طبية علمية صناعية غير مؤثرة على جسم الإنسان ومتوفرة في كل مكان.

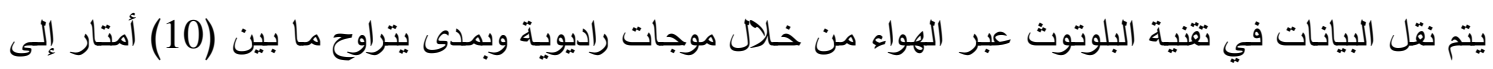
(100) متر بالاعتماد على الطاقة المتوفرة إذ كلما ازداد مدى الاتصال ازداد معه الطاقة المستهلكة، لذاء كانت هذهاء

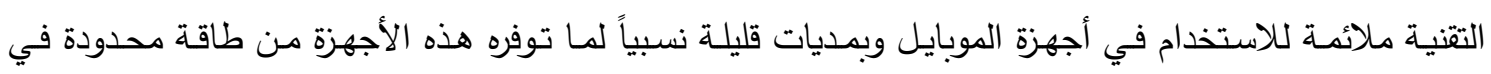
بطارياتها.

يتكون مكدس بروتوكول البلوتوث من جزأين أساسيين هـا مضيف البلوتوث Bluetooth Host ووحدة تحكم البلوتوث Bluetooth Controller، وتقدم واجهة التحكم المضيف Host Control Interface (HCI) واجهة هينة Bluetooth Controller ووحدة تحكم البلوتوث Bluetooth Host بين مضيف البلوتوث

وكما موضح في الثكل (2).
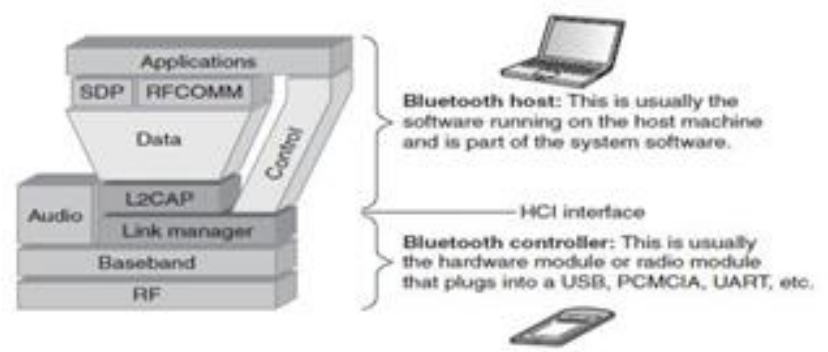

$$
\text { شكل (2) مكتّ بروتوكول البلوتوث بجزأيه : مضيف البلوتوث ووحدة تحكم البلوتوث [2] }
$$

يعرف مضيف البلوتوث Bluetooth Host بطبقات المكدس العليا Upper-Layer Stack والتي تتفذ عادة في

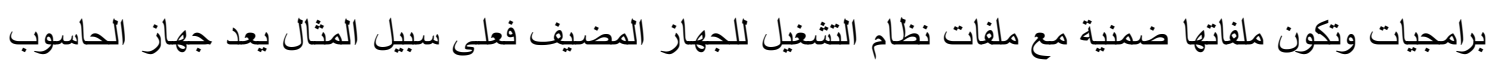
المحمول Laptop وجهاز الموبايل هو جهاز مضيف وتكون ملفات مضيف البلوتوث ضمنية داخل ملفات نظام التشغيل لجهاز الحاسوب المحمول أو لجهاز الموبايل.

أما وحدة تحكم البلوتوث Bluetooth Controller فهي عبارة عن قطعة مادية Hardware تضاف إلى الجهاز الذي سيجهز بخدمة البلوتوث، وممكن أن تكون هذا القطعة ضمنية Built in داخل القطع المادية للجهاز ، ويكون الربط بين وحدة التحكم مع نظام الجهاز المضيف عن طريق قنوات الإدخال والإخراج I/O Devices التي يوفرها

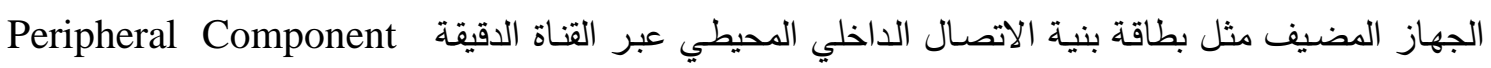
Micro Channel Interconnect Architecture (PCMCIA)

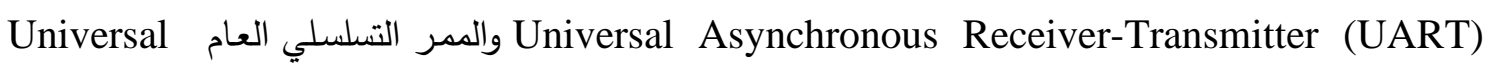
Serial Bus (USB) على الرغم لما ذكر أعلاه من تصنيف وحدة مضيف البلوتوث ووحدة التحكم يمكن أن يكون الاثنان في جهاز واحد

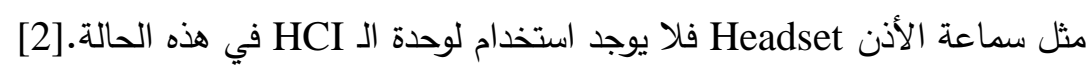

J2ME 3

تعد لغة Java 2 Micro Edition الثورة الثانية في التاريخ القصير للغة Java، عندما تم الإعلان عن لغة جافا

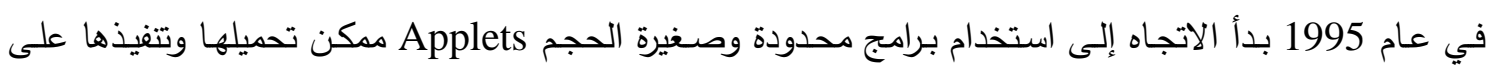
الأجهزة ومع تطور صناعة الأجهزة الصغيرة الحجم ازدادت أهمية لغة جافا لسببين : الأول إمكانية كتابة المقطع 
البرمجي من قبل المطورين Developers وتتفيذه على عشرات المنصـات Platforms وبدون تغيير ، والثاني امتلاك لغة جافا لخواص أمان عند تحميل المقطع البرمجي. لا تعد لغة J2ME مقطعاً أو مواصفات معينة وإنما هي كل ما تعنيه لغة جافا للأجهزة الصغيرة كأجهزة الهواتف

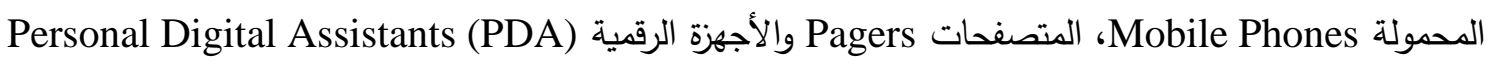

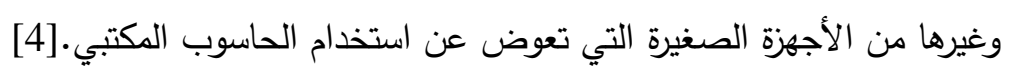

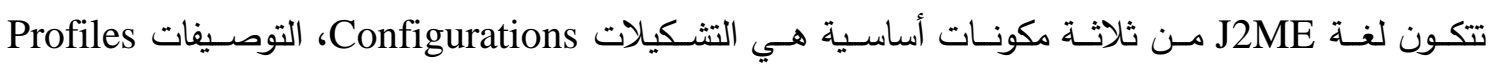
والواجهات البرمجية الاختيارية Application Programming Interface (APIs) وسيتم شرح كل مكون بإيجاز وكما يلي :

\section{3 التشكيلات Configurations}

صممت التشكيلات لنوع محدد من الأجهزة اعتماداً على حجم الذاكرة وطاقة المعالج للجهاز وتقوم بتحديد الآلّة

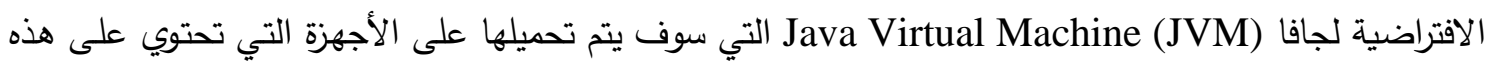

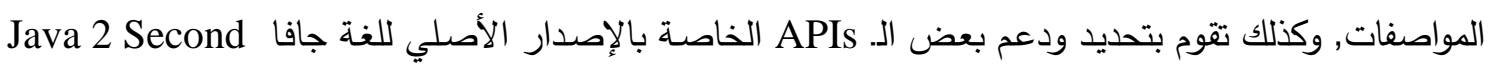
Edition J2SE

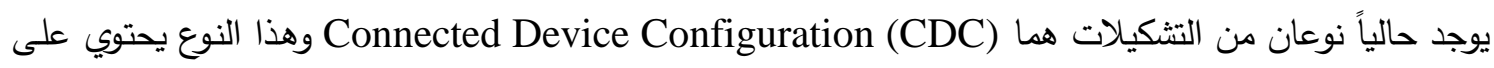

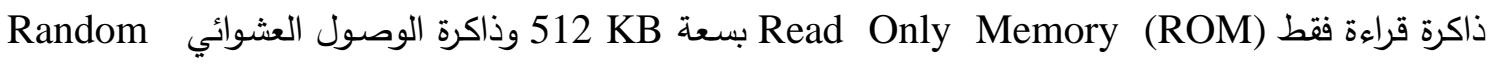
Access Memory (RAM) 256 KB على الأقل وكذلك بعض إمكانيات الاتصال بالثبكة. هذا النوع

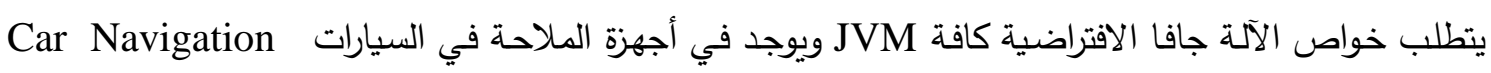
Systems والـ PDAs وغيرها.

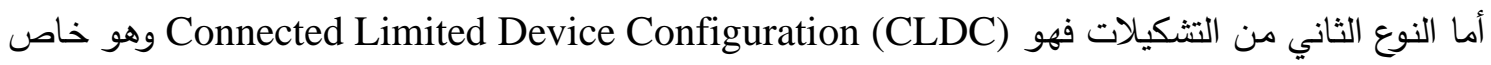
بالأجهز الصغيرة الحجم مثل الموبايل، الـ Pager والـ PDAs وغيرها من الأجهزة الصغيرة الحجم. ويحتوي هذا النوع على ذاكرة كلية صغيرة نسبياً تتراوح ما بين 512 KB 160 KB مع إمكانيات محدودة للاتصال بالثبكة التهرة

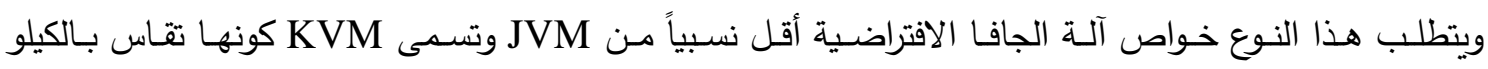
بايت.

\section{Profiles 2.3 التوصيفات}

وهي أكثر تحديداً من التشكيلات وتعتمد على التشكيلات، الـ APIs المضـافة، كمية الخزن الملائمة وأي تطبيق

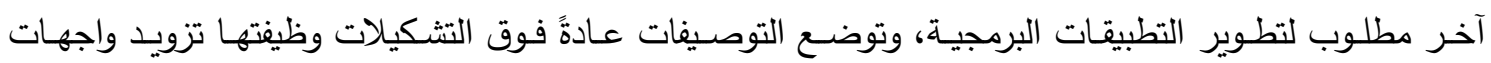
للوظائف التي يحتويها الجهاز وكذلك توفير بيئة الاتصال للشبكة.

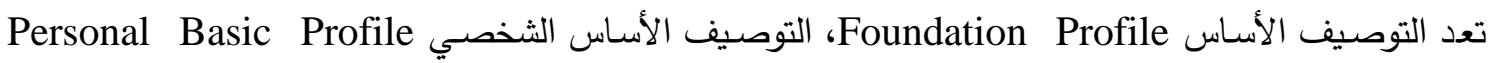
والتوصيف الثخصي Personal Profile من التوصيفات التي يوفرها الـ CDC والـ Personal Digital Assistant Profile (PDAP) [6].CLDC يوفرها الـ 
APIs 3.3

وهي مجموعة من الوظائف التي يتم إضـافتها إلى تثكيل معين ضمن حزمة مرنة، وتعد الـ CLDC هي نواة الـ

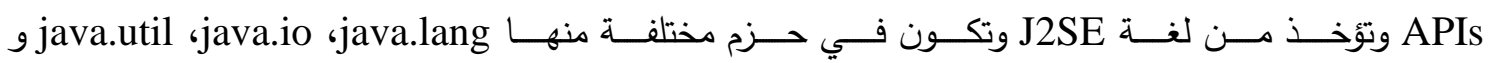

[6].J2ME java.microedition.io

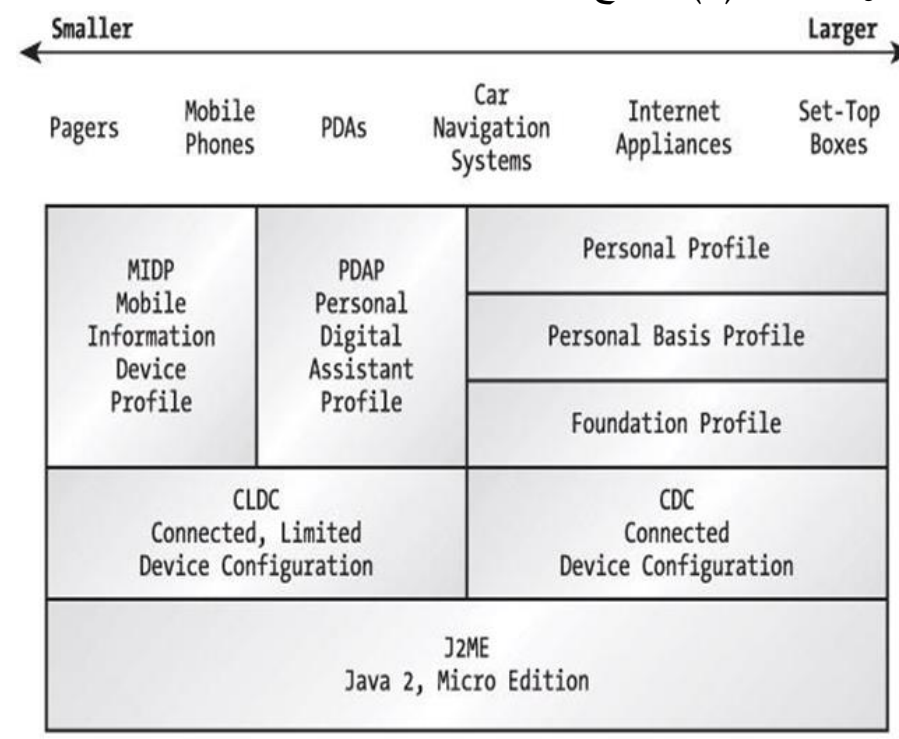

شكل (3) مكونات لغة J2ME [6]

4. - 20 - الأعمال السابقة

هنالك البعض من الأعمال السابقة في مجال استخدام تقنية البلوتوث في تتفيذ العديد من التطبيقات عن طريق الاتصال ما بين جهاز الموبايل وجهاز الحاسوب في البعض منها أو عن طريق الاتصال ما بين جهاز الموبايل

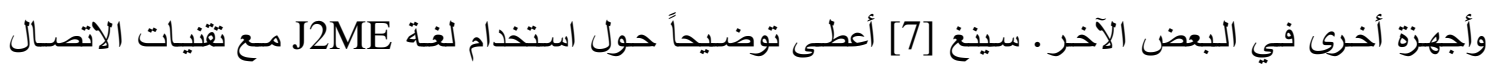
اللاسلكية (البلوتوث)، رؤيسة شاملةً حول التوصيفات Profiles، الصنوف المستخدمة Classes وكذلك الواجهات الاختيارية Application Programming Interface (APIs) مالفاتي Malfatti. [8 استخدم تقنية البلوتوث في التحكم ببعض الألعاب المطورة على جهاز الحاسوب، استخدم لغـة J2ME في برمجـة التطبيق مـع تقنيـة البلوتوث. آند ريد Andrade [9] استخدم لغة جافا مع تقنية البلوتوث في التحكم على لعبة السيارة من خـلال

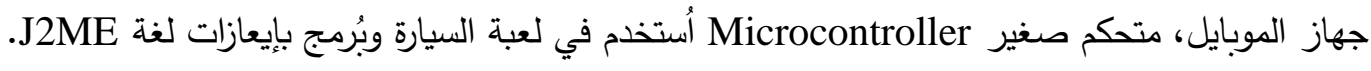
5. تطبيقات الموبايل العملية المقترحة في البحث :

تم في هذا البحث اقتراح وتتفيذ عدة برامج تطبيقية تعمل على أجهزة الموبايل وفيما يلي شرح التطبيقات المصممة في البحث.

1.5 تطبيق إدارة المكتبة الكترونياً Electronic Library Management Application

يقدم هذا التطبيق تسهيلاً واسعاً للشخص الذي ينوي الدخول الكترونياً إلى المكتبة من حيث البحث عن أي كتاب

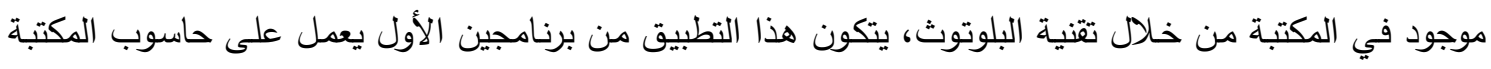

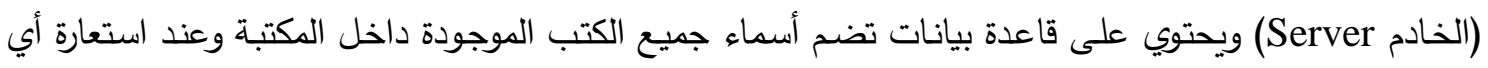
كتاب من قبل المستخدم سوف يقوم البرنامج بتحديث قاعدة البيانات وتاريخ إعادة الكتاب المستعار إلى المكتبة 
(والذي يحدد حسب الرغبة) فإذا بحث أي مستخدم آخر عن الكتاب الذي تم استعارته سوف يعلم بأن الكتاب

$$
\text { مستعار ويعلم التاريخ الواجب إعادة الكتاب فيه. }
$$

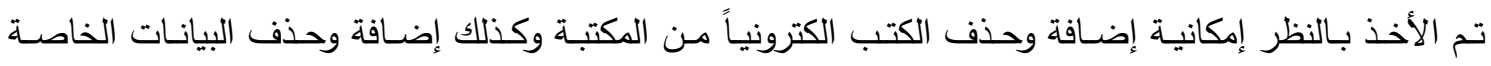
بالمستعيرين وحساب تاريخ إعادة الكتاب المستعار آلياً اعتماداً على تاريخ الاستعارة.

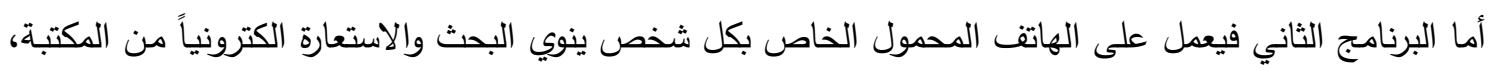

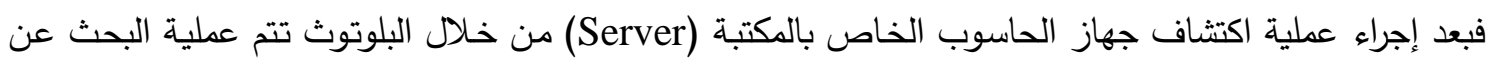
أي كتاب عن طريق إدخال اسم الكتاب أو اسم المؤلف أو الرقم التصنيفي (ID) الخاص بكل كتاب داخل المكتبة.

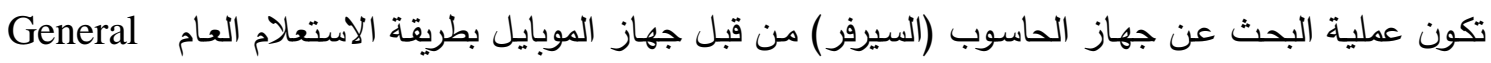

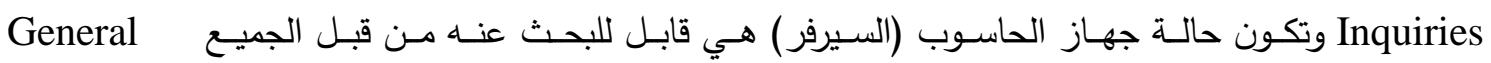
Discoverable، والثكل (4) يوضح المخطط الانسيابي لخوارزمية التطبيق المقترحة.

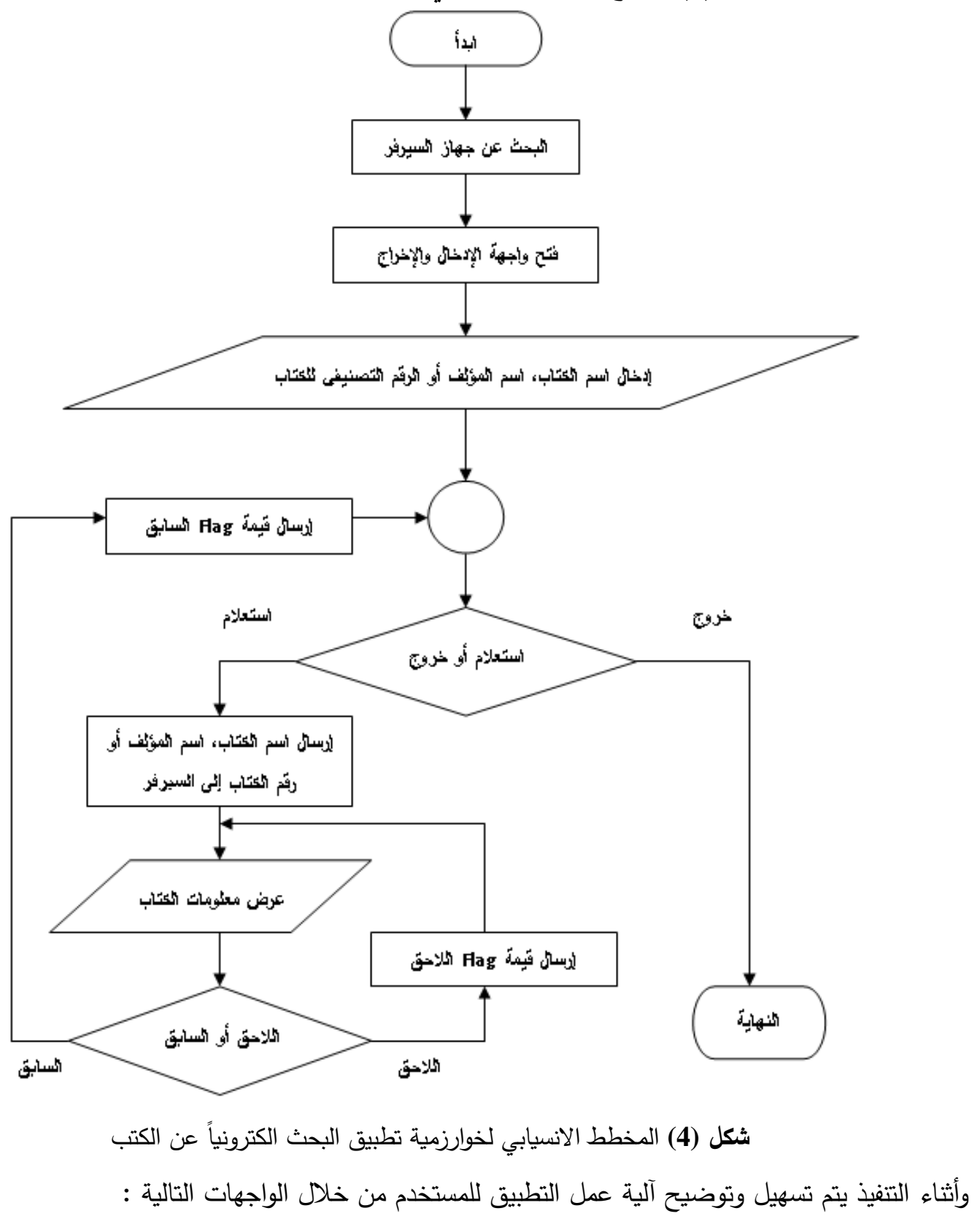




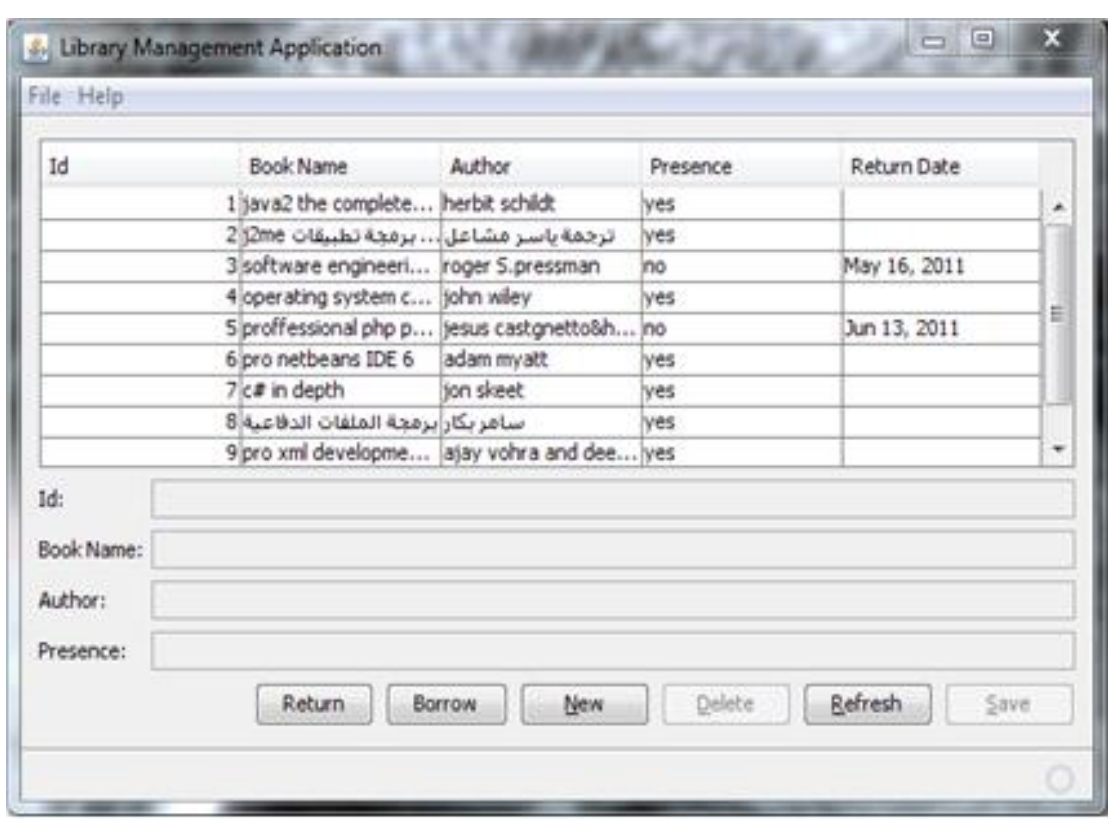

$$
\text { شكل (4) واجهة المعلومات عن الكتب }
$$

في الثكل (4) تظهر هذه الواجهة في برنامج الحاسوب الخادم ويتم من خلالها إضافة وحذف الكتب الكترونياً من المكتبـة. وبعد الضــط على زر Borrow تظهر الواجهة التاليـة كمـا في الثكل (6) الخاصـة بإدخال بيانـات الثخص المستعير ويتم من خلالها أيضـاً تثبيت تاريخ الاستعارة تبعاً لذلك تحدّث كافـة البيانـات داخل قاعدة

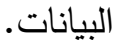

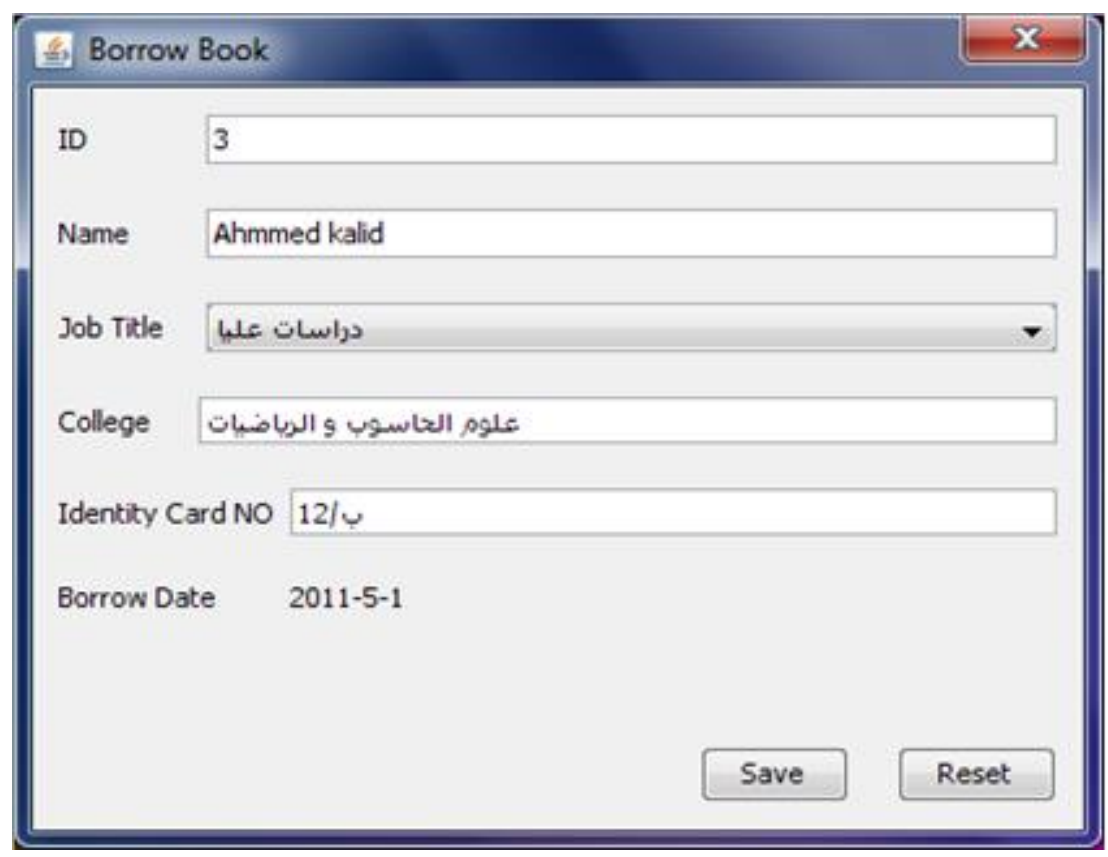

شكل (6) واجهة استعارة كتاب

الثكل (7) يبين أنه عند الضغط على زر Return في واجهة المعلومات عن الكتب (شكل (5)) تظهر الواجهة التالية المتعلقة بحذف البيانات الخاصة بالثخص المستعير بعد إعادة الكتاب المستعار . 


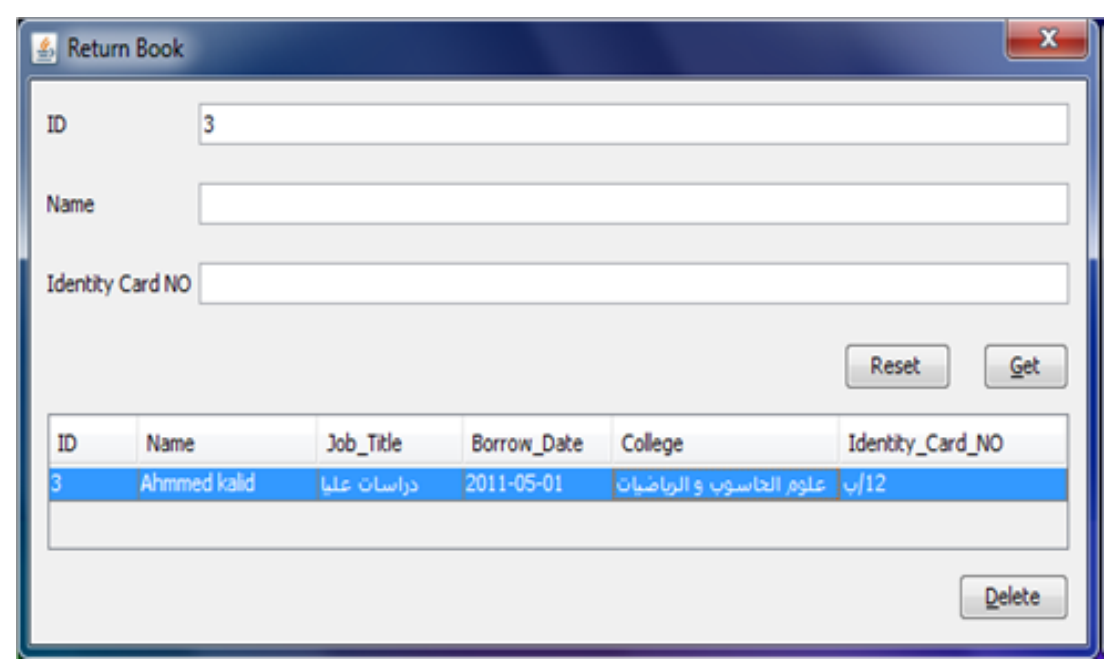

شكل (7) واجهة حذف البيانات

أمـا بالنسبة إلى جهاز الهاتف المحمول للشخص الذي ينوي الدخول إلى المكتبة الكترونياً فسوف تظهر فيـه الواجهات التي في الثكل (8)، إذ توضـح الواجهة (A) الأيقونـة الخاصـة بالتطبيق وتوضـح الواجهة (B) عمليـة

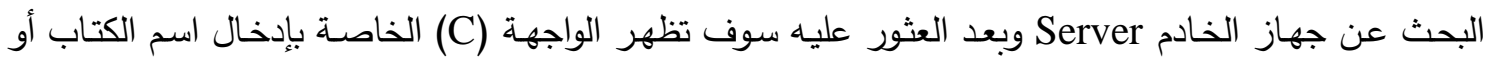

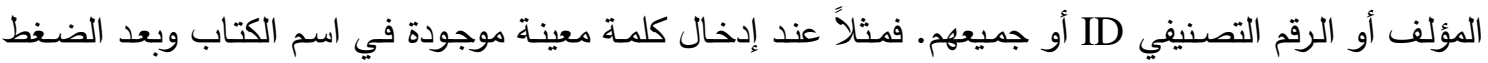
على Query سوف تظهر الواجهة (D) التي تظهر فيها معلومات الكتاب الذي يحتوي على هذه الكلمة في عنوانه وعند الضغط على Next سوف تظهر معلومات خاصة بكتاب آخر في قاعدة البيانات يحتوي على الكلمة نفسها في عنوانه وهكذا. تم كتابة برنـامج الحاسوب الخادم باستخدام لغـة J2SE وهي من اللغات التي تدعم التعامل مـع قواعد البيانات

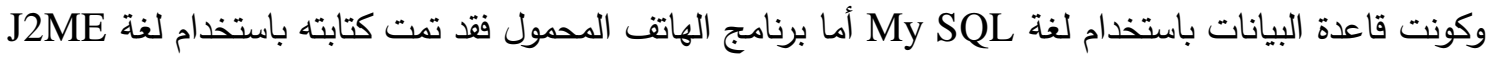
ونشر البرنامج على منصة 2.0 و MIDP و CLDC 1.0 وظهرت النتائج صحيحة بدون أخطاء. أُعيد تشغيل

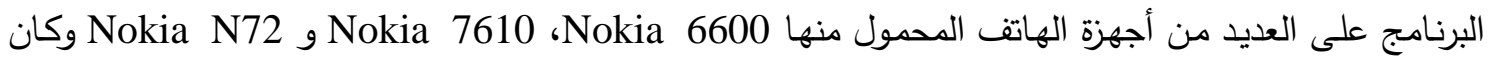
التتفيذ بصورة صحيحة على جميع هذه الأجهزة.

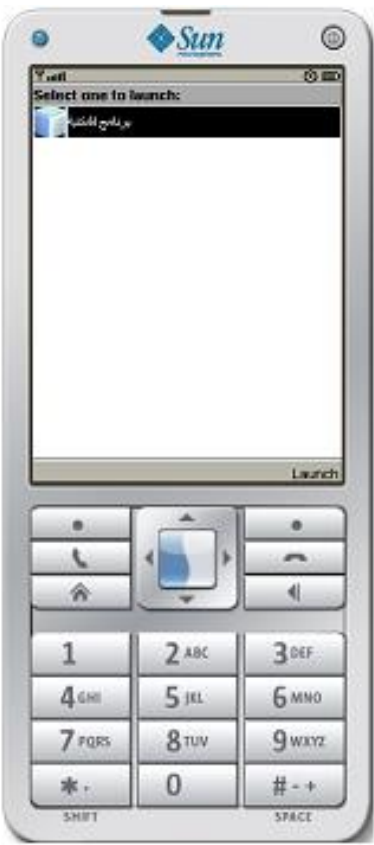

(A)

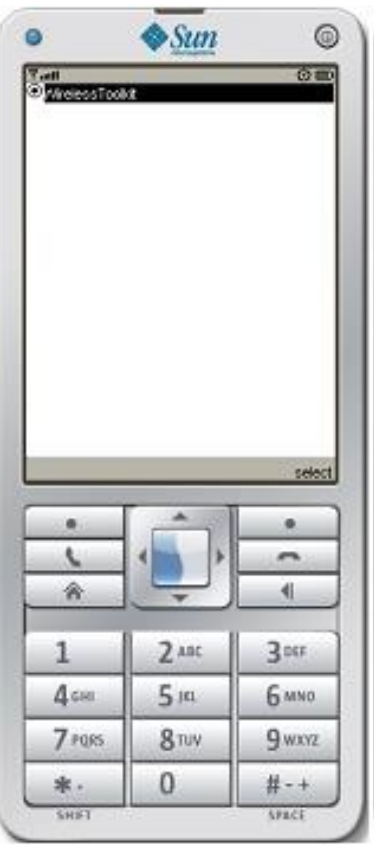

(B)

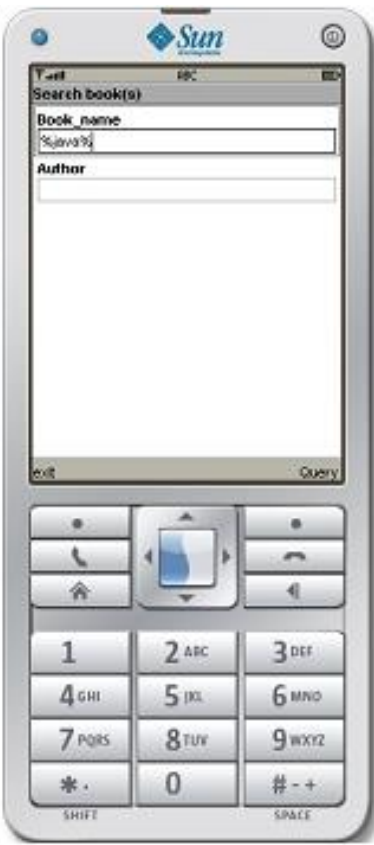

(C) 


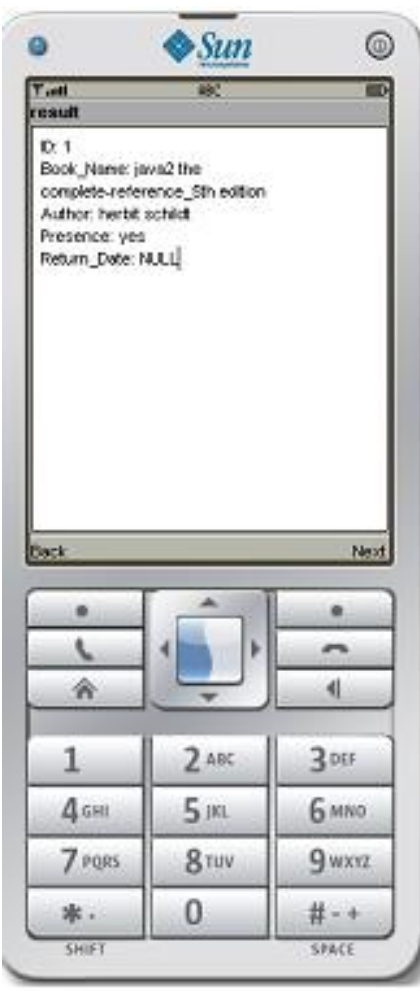

(D)

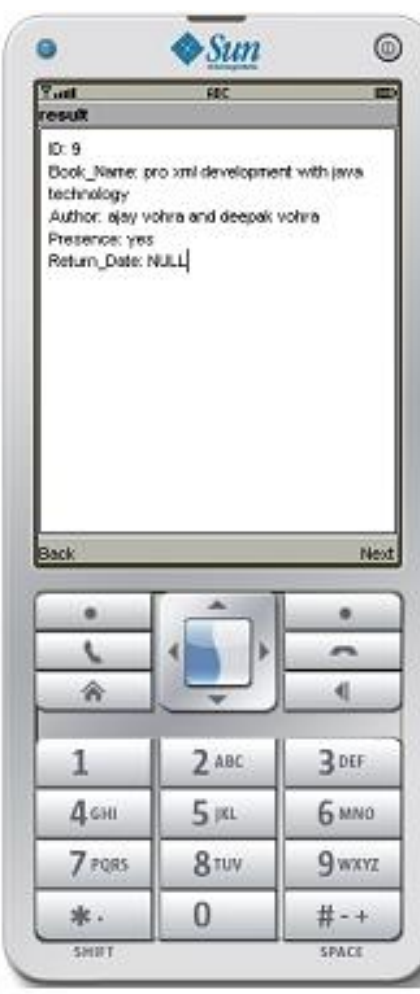

(E)

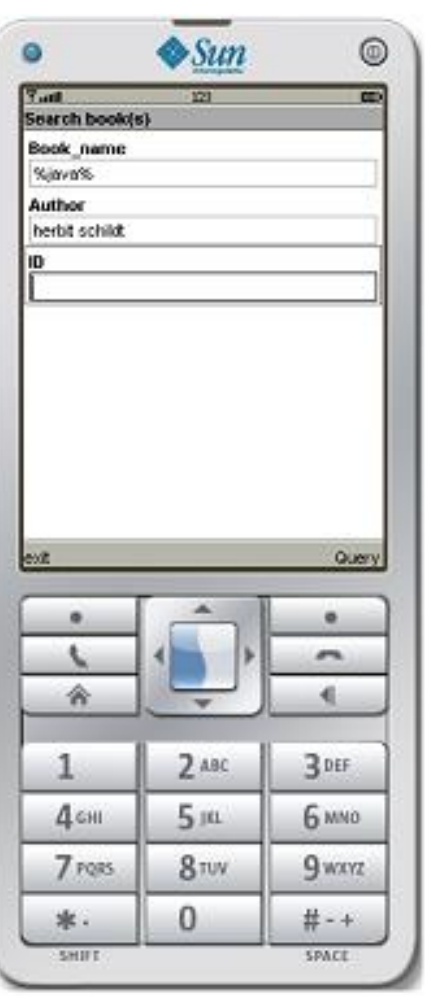

(F)

شكل (8) واجهات الهاتف المحمول بتطبيق إدارة الدكتبة الكترونياً

2.5

يقوم هذا التطبيق بتوفير إمكانية التحكم بفأرة الحاسوب ( Mouse ) باستخدام الهاتف المحمول عن طريق تقنية البلوتوث حيث تكمن فائدة هذا التطبيق في استعماله وسيلة تعليمية للتدريسيين والمدربين دون اللجوء إلى استخدام الماوس خلال عملية عرض الشرائح لذا فقد تم استخدام هذا التطبيق والاستفادة منه في مجالين عمليين الأول هو التحكم بمؤشر الماوس والثاني هو التحكم بعرض الشرائح على الحاسوب. يتكون هذا التطبيق من برنامجين الأول يعمل على جهاز الهاتف المحمول والثاني يعمل على جهاز الحاسوب، يقوم البرنامج الأول بإرسال طلب لتغيير موقع مؤشر الماوس في جهاز الحاسوب إلى إحداثي موقع جديد حيث يقوم البرنامج الثاني بتحليل الطلب القادم من البرنامج الأول ومن ثم تتفيذه على الحاسوب. تكون عملية البحث عن جهاز الحاسوب من قبل جهاز الموبايل هي نفسها في تطبيق إدارة المكتبة الكترونياً أي بطريقة الاستعلام العام General Inquiries وتكون حالـة جهاز الحاسوب هي قابـل للبحث عنـه مـن قبل الجميع General Discoverable، ويوضـح الثكل (9) المخطط الانسيابي لخوارزمية عمل التطبيق : 


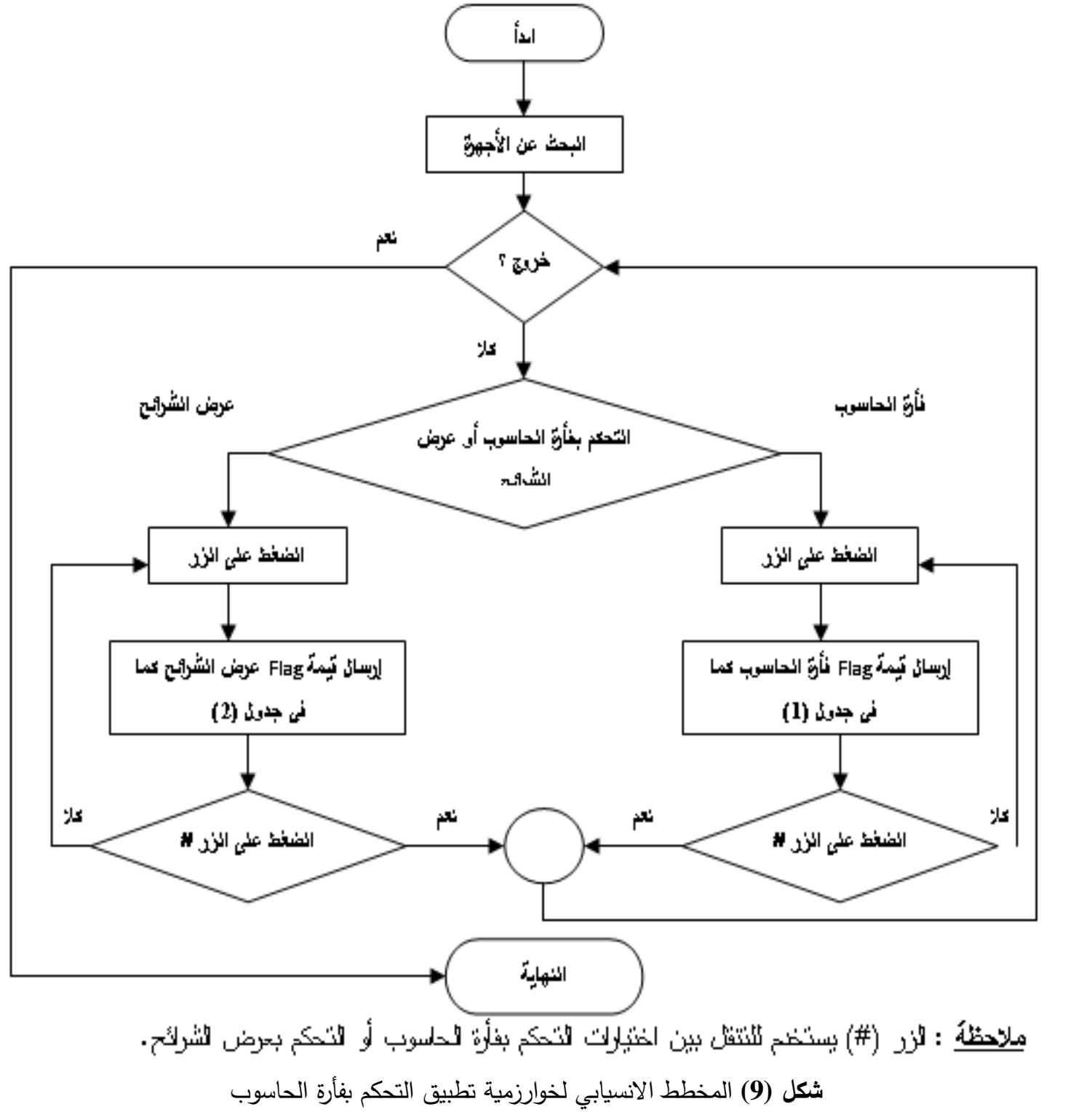

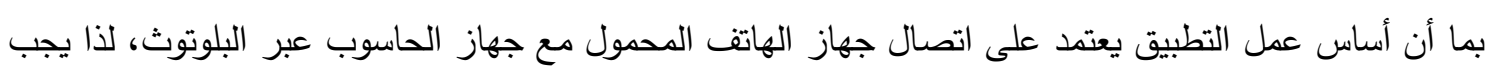
تقعيل الخدمة من قبل جهاز الحاسوب. يقوم برنامج التطبيق الخاص بجهاز الحاسوب بعرض واجهة خاصة لبدء عرض الاتصال ويقوم برنامج التطبيق الخاص بالهاتف المحمول بتوفير القابلية على البحث على الأجهزة المحيطة وعرضها في قائمسة ليتسنى للمستخدم تحديد الجهاز المطلوب الاتصـال معه. بعد تشغيل البرنامج تظهر الواجهة

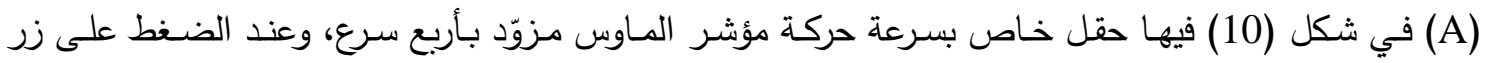
Connect لإجراء الاتصال وبعد إجراء الاتصال تظهر الواجهة (C) الخاصة بقطع الاتصال بين الجهازين. 


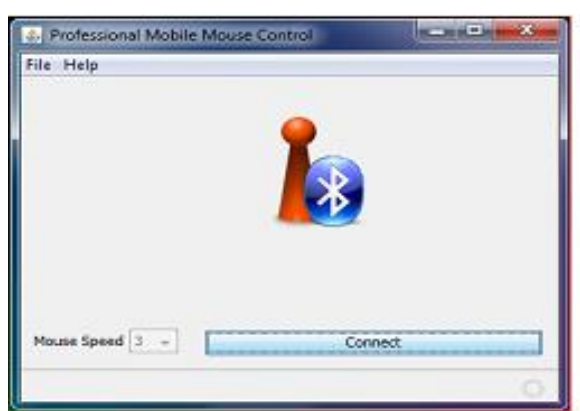

(A)

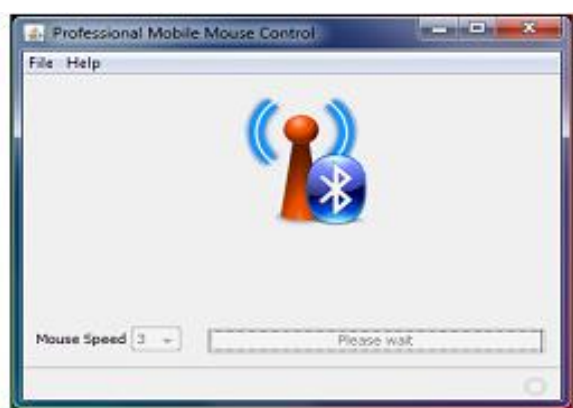

(B)

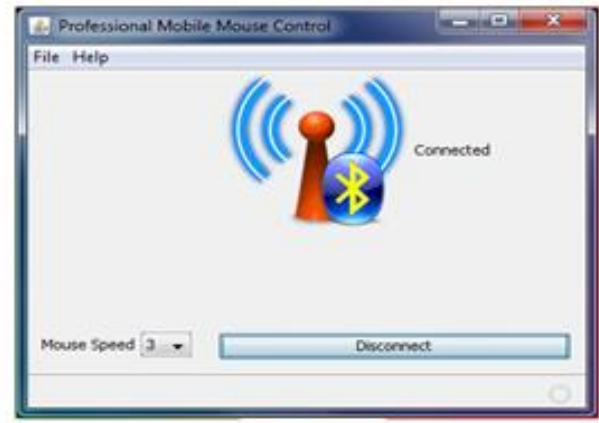

(C)

شكل (10) واجهات الاتصال عبر البلوتوث

أما بالنسبة لواجهات الهاتف المحمول المبينة في الثكل (11)، توضـح الواجهة (A) الأيقونة الخاصـة بالتطبيق وبعد تشغيل التطبيق تظهر الواجهة (B) الخاصـة بعرض الأجهزة المحيطة على شكل قائمة. بعد اختيار الجهاز المطلوب الاتصـال معـه تظهر الواجهة (C) ويتم فيها تحديد الاختيار سواءً كان التحكم بفأرة الحاسوب أم التحكم بعرض الشرائح. إذا كان الاختيار الأول سوف تظهر الواجهة (D)، عند الضغط على الزهل الزر المتواجد في وسط الاتجاهـات سوف يقوم البرنـامج بضغط نقرة واحدة عند الاحداثي الحسالي، عند الضـغط على الأمـر R-Click الموجود أسفل الواجهة إلى اليمين سيقوم البرنامج بضغط نقرة اليمين، عند الضغط على أمر Click-On الموجود أسفل الواجهة إلى اليسار سيقوم البرنامج بالضغط المستمر على نقرة الماوس في الاحداثي الحالي.

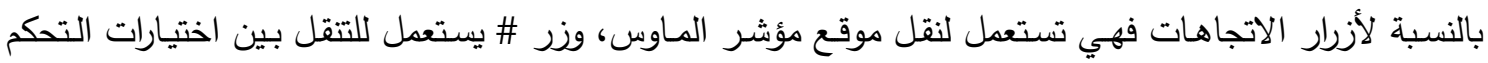
بالماوس والتحكم بعرض الثرائح. أما إذا كان الاختيار الثاني وهو التحكم بعرض الثرائح المبين في الواجهة (C)

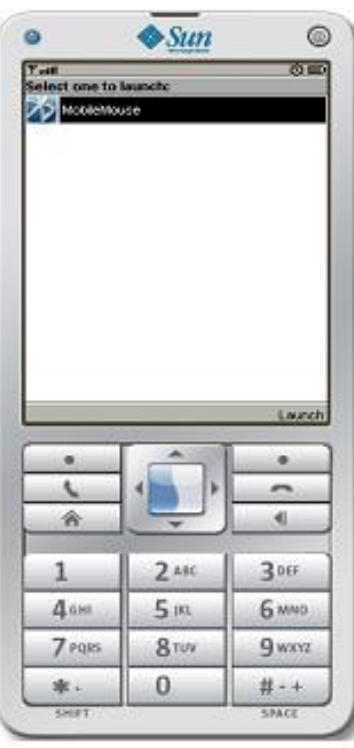

(A)

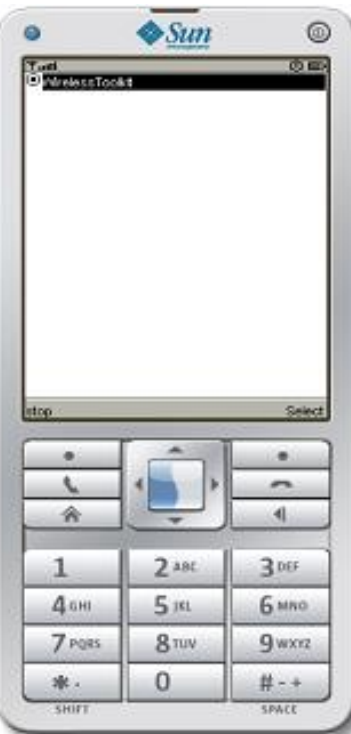

(B)

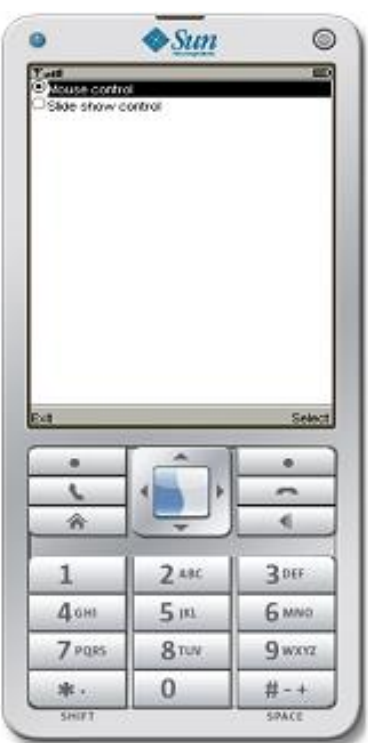

(C) في الواجهة 


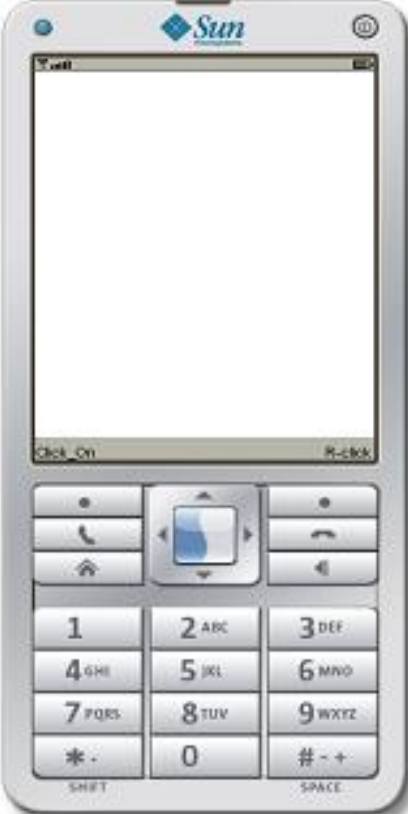

(D)

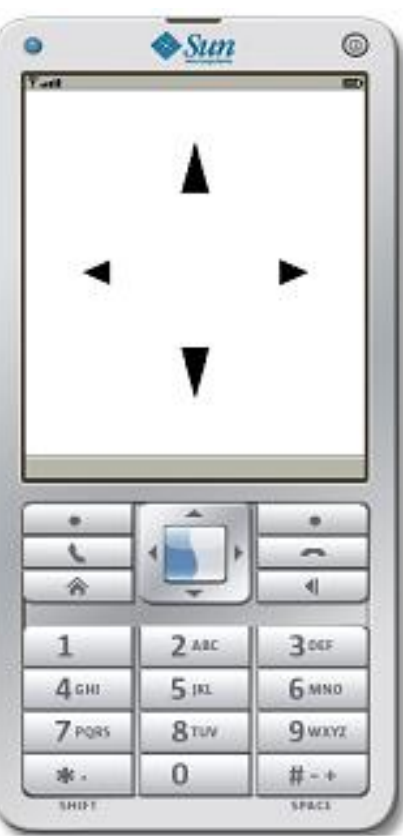

(E)

شكل (11) واجهات الموبايل بعد تشغيل تطبيق التحكم بفأرة الحاسوب

الثالثة فسوف تظهر الواجهة (E) الخاصـة بالتحكم بعرض الثرائح. عند الضغط على أزرار الاتجاهات فسيكون تتفيذ مفاتيح الاتجاهات على جهاز الحاسوب للتنقل بين الشرائح. يوضح الجدولان (1) و (2) الوظائف التي يؤديها كل زر من أزرار الهاتف المحمول في التحكم بالماوس وعرض بـ

جدول Mouse Flag (1)

\begin{tabular}{|c|c|}
\hline Flag Value & Meaning \\
\hline 0 & Click on \\
\hline 1 & Click off \\
\hline 2 & Right click \\
\hline 3 & Move Mouse up \\
\hline 4 & Move Mouse down \\
\hline 5 & Move Mouse left \\
\hline 6 & Move Mouse right \\
\hline 7 & Wheel up \\
\hline 8 & Wheel down \\
\hline 9 & Exit program \\
\hline
\end{tabular}

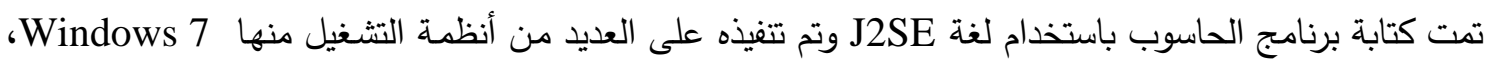

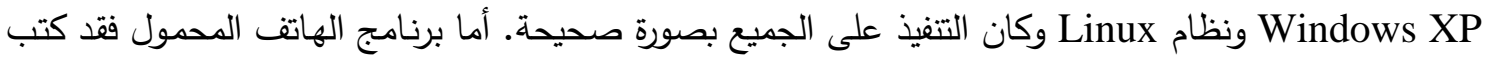

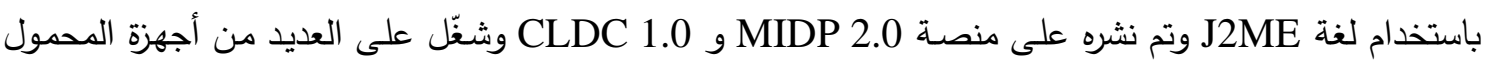
منها Nokia N72، Nokia 7610 و Nokia 6600 وكانت النتائج صحيحة للجميع. 


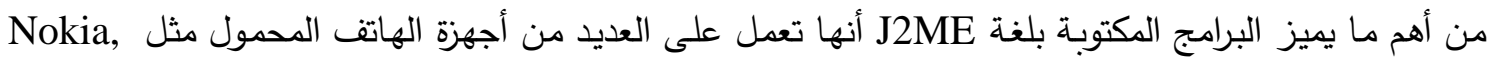

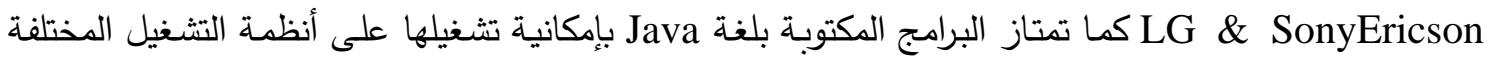
مثل Windows و Linux بالنسبة لتطبيق إدارة المكتبة الكترونياً فيوفر تسهيلات كبيرة عن طريق استخدام أجهزة الموبايل في البحث عن

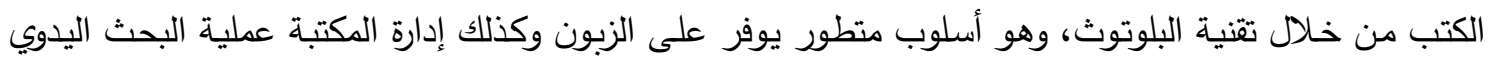

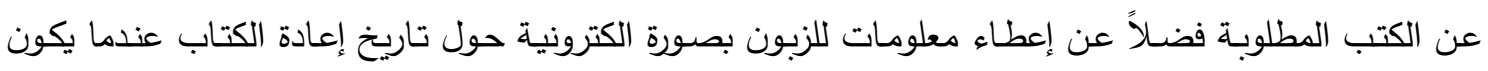
الكتاب المطلوب مستعاراً. أما بالنسبة لتطبيق التحكم بفأرة الحاسوب فيمكّن المستخدم من التعامل مع الحاسوب عن بعد وبدون الحاجة إلى الفأرة وذلك عن طريق تقنية البلوتوث وباستخدام جهاز الموبايل. ومن الفوائد التي يقدمها هذا التطبيق إمكانيـة

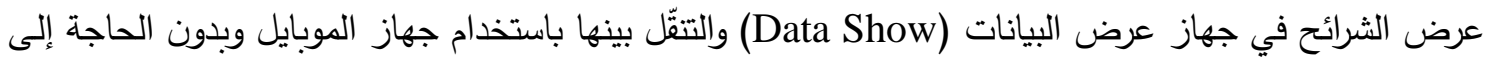
ضرورة التواجد عن قرب من جهاز الحاسوب المستخدم.

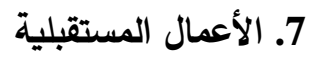
يمكن تطوير تطبيق المكتبة من خلال إضافة إمكانية عرض ملخص الكتاب والمحتويات للكتاب المطلوب استعارته

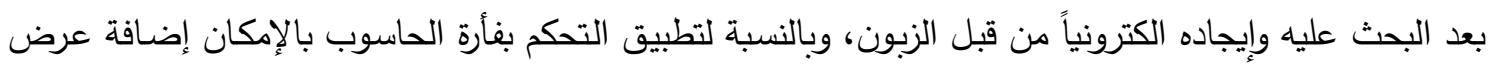
صورة موقع المؤشر الحالي للفأرة. 


\section{المصادر}

[1] Mallick M., 2003, "Mobile and Wireless Design Essentials", Wiley Publishing, Inc., Indianapolis, Indiana.

[2] Kumar C. B., 2004, "Bluetooth Application Programming with the Java APIs", Morgan Kaufmann, San Francisco.

[3] Fling B., 2009, "Mobile Design and Development", O’Reilly Media.

[4] Knudsen J., 2005, "Beginning J2ME: From Novice to Professional", Third Edition, APress.

[5] Jode M. D., 2004, "Programming Java 2 Micro Edition on Symbian OS", Wiley.

[6] Knudsen J., 2003, "Wireless Java Developing with J2ME", Second Edition, APress.

[7] Singh P., 2011, " Study of Bluetooth Wireless Technology Using Java", Indian Journal of Computer Science and Engineering (IJCSE).

[8] Malfatti S. M., 2010, " Using Mobile Phones to Control Desktop Multiplayer Games", Brazilian Symposium on Computer Games and Digital Entertain.

[9] Andrade F. S., 2011, " General Purpose Bluetooth Control", IEEE Latin America Transactions. 